\title{
A Web-Based Prediction Model for Cancer-Specific Survival of Elderly Patients With Clear Cell Renal Cell Carcinoma: A Population-Based Study
}

\author{
Chenghao Zhanghuang ${ }^{1,2}$, Jinkui Wang ${ }^{1}$, Zhaoxia Zhang ${ }^{1}$, Liming Jin ${ }^{1}$, Xiaojun Tan ${ }^{1,3}$, \\ Tao Mi ${ }^{1}$, Jiayan Liu ${ }^{1}$, Mujie $\mathrm{Li}^{1}$ and Dawei He ${ }^{1 *}$

\begin{abstract}
' Department of Urology, Chongqing Key Laboratory of Children Urogenital Development and Tissue Engineering, Chongqing Key Laboratory of Pediatrics, Ministry of Education Key Laboratory of Child Development and Disorders, National Clinical Research Center for Child Health and Disorders, China International Science and Technology Cooperation Base of Child Development and Critical Disorders, Children's Hospital of Chongqing Medical University, Chongqing, China, ${ }^{2}$ Yunnan Key Laboratory of Children's Major Disease Research, Department of Urology, Kunming Children's Hospital (Children's Hospital Affiliated to Kunming Medical University), Kunming, China, ${ }^{3}$ Department of Urology, Nanchong Central Hospital, The Second
\end{abstract} \\ Clinical Medical College, North Sichuan Medical University, Nanchong, China
}

OPEN ACCESS

Edited by:

Thippa Reddy Gadekallu, VIT University, India

Reviewed by: Abdul Rehman Javed, Air University, Pakistan Sharnil Pandya, Symbiosis International

University, India

*Correspondence:

Dawei He

hedawei@hospital.cqmu.edu.cn

Specialty section:

This article was submitted to

Digital Public Health,

a section of the journal

Frontiers in Public Health

Received: 12 December 2021 Accepted: 28 December 2021 Published: 03 March 2022

Citation:

Zhanghuang C, Wang J, Zhang Z, Jin L, Tan X, Mi T, Liu J, Li M and He D

(2022) A Web-Based Prediction Model for Cancer-Specific Survival of Elderly Patients With Clear Cell Renal Cell Carcinoma: A Population-Based Study. Front. Public Health 9:833970.

doi: 10.3389/fpubh.2021.833970
Background: Clear cell renal cell carcinoma (cCRCC) is expected in the elderly and poor prognosis. We aim to explore prognostic factors of ccRCC in the elderly and construct a nomogram to predict cancer-specific survival (CSS) in elderly patients with ccRCC.

Methods: Clinicopathological information for all elderly patients with ccRCC from 2004 to 2018 was downloaded from the Surveillance, Epidemiology, and End Results (SEER) program. All patients were randomly assigned to a training cohort $(70 \%)$ or a validation cohort (30\%). Univariate and multivariate Cox regression models were used to identify the independent risk factors for CSS. A new nomogram was constructed to predict CSS at 1-, 3-, and 5 years in elderly patients with ccRCC based on independent risk factors. Subsequently, we used the consistency index (C-index), calibration curves, and the area under the receiver operating curve (AUC) and decision curve analysis (DCA) to test the prediction accuracy of the model.

Results: A total of 33,509 elderly patients with ccRCC were enrolled. Univariate and multivariate Cox regression analyses results showed that age, sex, race, marriage, tumor size, histological grade, tumor, nodes, and metastases (TNM) stage, and surgery were independent risk factors for CSS in elderly patients with ccRCC. We constructed a nomogram to predict CSS in elderly patients with cCRCC. The C-index of the training cohort and validation cohort was 0.81 (95\% Cl: 0.802-0.818) and 0.818 (95\% Cl: $0.806-0.830)$, respectively. The AUC of the training cohort and validation cohort also suggested that the prediction model had good accuracy. The calibration curve showed that the observed value of the prediction model was highly consistent with the predicted value. DCA showed good clinical application value of the nomogram.

Conclusion: In this study, we explored prognostic factors in elderly patients with ccRCC. We found that age, sex, marriage, TNM stage, surgery, and tumor size were independent 
risk factors for CSS. We constructed a new nomogram to predict CSS in elderly patients with ccRCC with good accuracy and reliability, providing clinical guidance for patients and physicians.

Keywords: nomogram, clear cell renal cell carcinoma, cancer-specific survival, elderly patients, SEER

\section{INTRODUCTION}

Renal cell carcinoma (RCC) is a malignant tumor originating from renal tubular epithelial cells. More than 400,000 cases are diagnosed each year, and RCC incidence is about twice as high in men as in women $(1,2)$. There are several major pathological subtypes of RCC, of which clear cell renal cell carcinoma (ccRCC) accounts for $70-80 \%$, with papillary renal cell carcinoma (PRCC) and chromophobe renal cell carcinoma (CRCC) remaining $(3,4)$. ccRCC is characterized by rich glycogen and lipids in cells and is manifested as chromosomal $3 p$ deletion and genomic mutation in Von Hippel-Lindau (VHL) tumor suppressor allele (5). Although ccRCC is a disease that can be cured by early surgery, such as radical nephrectomy $(\mathrm{RN})$, partial nephrectomy (PN), and local tumor excision represented by radiofrequency ablation (RFA), recurrence and metastasis occur in up to one-third of cases (6). Moreover, these metastases often suggest poor prognosis, which makes the prognosis of ccRCC significantly different from that of other RCCs (7).

Age is a significant risk factor for cancer. With the increase of age, the probability of cell mutation caused by molecular changes also increases, and cell mutation is generally considered the initiation event of cancer (8). The elderly over 65 years old account for more than $75 \%$ of the diagnosed patients with ccRCC. With the aging population and the extension of life expectancy, the incidence rate of renal cancer in the elderly is still increasing year by year (2).

Unfortunately, until today, studies on genetics (9), molecular structure (10), and clinicopathological features (11) have not been able to predict the clinical outcome and prognosis of ccRCC patients accurately. Accurate prediction is crucial in clinical decision-making, patient confidence building, and medical care improvement. In recent years, the application of nomogram prediction models, such as UISS (12), the Stage, Size, Grade, and Necrosis (SSIGN) (13), and Leibovich (14), in the prognosis of cancer patients provides clinicians with a basis for precise and individualized treatment of various cancer patients.

At present, artificial intelligence has been widely used in human health. Awais et al. (15) used texture analysis techniques in fluorescence imaging to help dentists identify areas of oncogenic cavity abnormalities in the oral cavity, and thus, perform biopsies more efficiently to predict the histological diagnosis of epithelial dysplasia. Shah et al. (16) used machine learning to detect the condition and risk of cardiac arrest early to improve survival in patients with heart disease.

However, to our knowledge, no predictive model for cancerspecific survival (CSS) of elderly ccRCC has been reported.
Moreover, the prognostic factors of survival in elderly patients with ccRCC remain unclear. We used big data from the Surveillance, Epidemiology, and End Results (SEER) program for the first time to explore factors influencing CSS in elderly patients with ccRCC. Based on the above situation, we developed the nomogram prediction model. We validated its accuracy in evaluating the CSS rate of patients with ccRCC, providing a reference for clinical diagnosis and treatment.

\section{PATIENTS AND METHODS}

\section{Data Source and Data Extraction}

We downloaded clinicopathological information for all elderly patients with ccRCC from 2004 to 2018 from the SEER program of the National Cancer Institute. SEER database is the National cancer database of the United States, covering about $30 \%$ of the population and containing 18 cancer registries. Demographic information, clinicopathological information, and follow-up data for all cancer patients are publicly available from the SEER database. Because patient data are publicly available and cannot be identified, our study needs not require ethical approval or informed consent from patients. Our research methods comply with the regulations of the SEER database.

All the demographic information of ccRCC in elderly patients (age, sex, race, year of diagnosis, and marriage), clinical pathologic information (laterality, tumor size, histological grade, and tumor, nodes, and metastases [TNM] stage), treatment (surgery, radiation therapy, and chemotherapy), follow-up information (survival status, survival time, and the cause of death) were collected. Inclusion criteria were as follows: (1) pathological diagnosis of ccRCC (International Classification of Diseases for Oncology [ICD-O]-3 codes, 8310); (2) age $\geq 65$; (3) the years of diagnosis were 2004-2018; and (4) unilateral renal tumor. Exclusion criteria were as follows:(1) tumor histological grade is unknown; (2) tumor size is unknown; (3) unknown surgical method; (4) survival time $<1$ month; and (5) incomplete follow-up information. The patient screening flow chart is shown in Figure 1.

The patients' race was divided into three categories: white, black, and others (American Indian/AK Native, Asian/Pacific Islander). Patients were divided into years of diagnosis from 2004 to 2010 and 2011 to 2018. The patient's tumor histological grades included grade I (welldifferentiated), grade II (moderately differentiated), grade III (poorly differentiated), and grade IV (undifferentiated). Patients were classified as non-operative (SEER Operation Code 0), local tumor excision (SEER Operation Code 10-27), PN (SEER Operation Code 30), and RN (SEER Operation Code 40-80). 


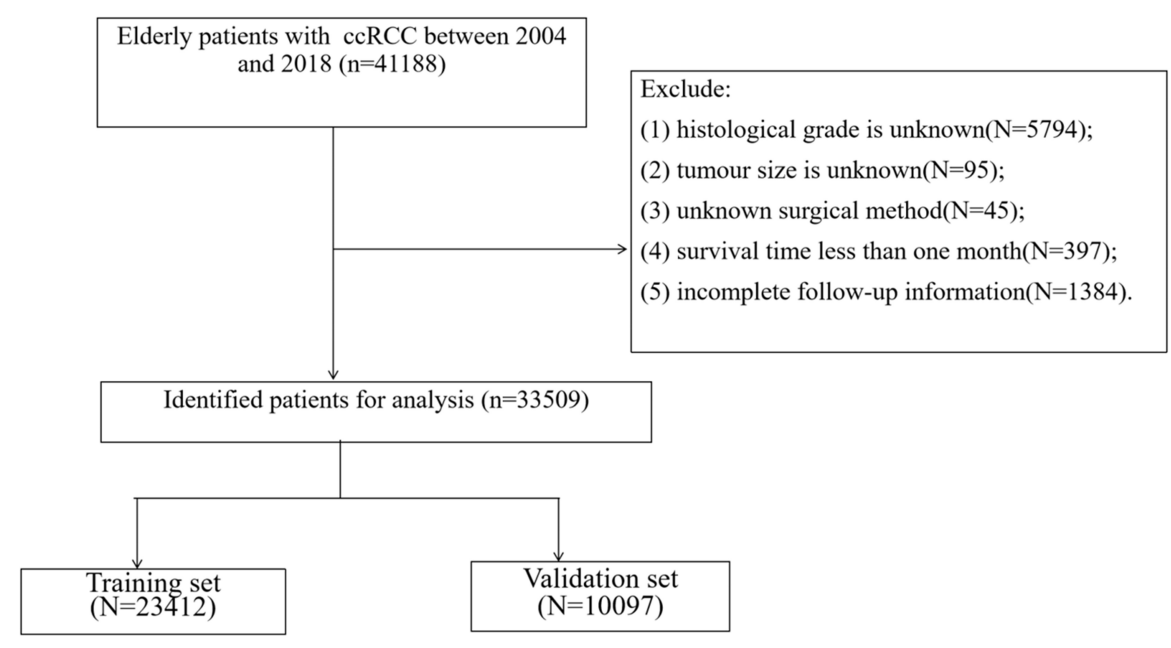

FIGURE 1 | Flowchart for inclusion and exclusion of all patients.

\section{Univariate and Multivariate Cox Regression Analyses}

All elderly patients with ccRCC were randomly assigned to a training cohort $(70 \%)$ or a validation cohort $(30 \%)$. All variables that might be associated with patient outcomes were included in the analysis. Univariate Cox regression models were used in the training cohort to analyze risk factors associated with in patients with CSS. According to univariate Cox regression analysis, a multivariate Cox regression model was established to identify the independent risk factors for CSS.

\section{Construction of Nomogram}

The establishment of the nomogram mainly relies on the multivariate Cox regression model. We developed a new nomogram to predict CSS at 1-, 3-, and 5 years in elderly patients with ccRCC based on independent risk factors for univariate and multivariate screening. Because these independent risk factors are predictors of patient outcomes.

\section{Validation of Nomogram}

Subsequently, we used the consistency index (C-index) to test the accuracy and discrimination of the prediction model in the training cohort and validation cohort. Calibration curves of 1,000 bootstrap samples were used to test the accuracy of the prediction model. The area under the receiver operating curve (AUC) was used to test the model's prediction accuracy at 1-, 3-, and 5 years.

\section{Clinical Utility}

We used the decision analysis curve (DCA) to validate the clinical potential of the predictive model. DCA is a new algorithm for calculating net benefit under different thresholds and is currently most commonly used to evaluate the clinical application value of models. In addition, we divided patients into high-risk and low-risk groups based on each patient's nomogram score and cutoff values determined by the receiver operating curve. Logrank test and Kaplan-Meier (K-M) curve were used to compare the survival difference between the two groups. In addition, we analyzed surgical differences between high-risk and low-risk patients based on risk grouping.

\section{Statistical Analysis}

Continuous measurement data were described by means and variance, and comparison between groups was performed by Chi-square test or non-parametric $U$ test. Count data were characterized by frequency (\%), and a chi-square test was used to compare groups. Univariate and multivariate Cox proportional regression models analyzed the survival and prognostic factors. Log-rank test and Kaplan-Meier (K-M) curve were used to analyze the survival differences between the patients. SPSS 26.0 and R Software 4.1 .0 were used for all statistical analyses. Values of $p<0.05$ were considered statistically significant.

\section{RESULTS}

\section{Clinical Features}

A total of 33,509 elderly patients with ccRCC were enrolled. All patients were randomly divided into the training cohort $(\mathrm{N}=$ $23,412)$ and the validation cohort $(\mathrm{N}=10,097)$. The mean age of all patients was $72.7 \pm 5.91$ years, 29,034 (86.6\%) white, 20,201 (60.3\%) men, and 21,239 (63.4\%) married. Among all patients, there were 16,447 (49.1\%) tumors on the left side, 4,077 (12.2\%) tumors at grade I, 17,480 (52.2\%) tumors at grade II, 9,595 $(28.6 \%)$ tumors at grade III, and 2,357 (7.03\%) tumors at grade IV. The mean tumor size was $51.8 \pm 32.3 \mathrm{~mm}$, the T stage T1a had $14,019(41.8 \%)$, T1b had 8,063 (24.1\%), T2 had 2,987 (8.91\%), T3 had 8,370 (25.0\%), and T4 had $70(0.21 \%)$. There were 32,620 (97.3\%) patients in stage N0 and 31,320 (93.5\%) patients in stage M0. There were 1,227 (3.66\%) patients without surgery, 1,718 (5.13\%) patients with local tumor excision, 8,791 (26.2\%) patients with PN, and 21,773 (65.0\%) patients with RN. There were 1,505 (4.49\%) patients who received chemotherapy and $640(1.91 \%)$ patients who received radiotherapy. The clinicopathological 
information of the patients is shown in Table 1, and there was no significant difference between the training cohort and the validation cohort.

\section{Univariate and Multivariate Cox Regression Analyses}

Univariate Cox regression model was used to analyze all variables to screen for variables associated with CSS in elderly patients with ccRCC. Age, sex, race, marriage, year of diagnosis, tumor size, histological grade, TNM stage, and surgery were prognostic factors related to patient survival. We included these factors in a multivariate Cox regression analysis. We found that age, sex, race, marriage, tumor size, histological grade, TNM stage, and surgery were independent risk factors for CSS in elderly patients with ccRCC. In other words, these independent risk factors can be used as factors in predictive models to predict CSS of the patients. The univariate and multivariate analyses results are shown in Table 2.

\section{Nomogram Construction for 1-, 3-, and 5-Year CSS}

We constructed a nomogram to predict CSS in elderly patients with ccRCC (Figure 2). Tumor size was the most significant risk factor for in patients with CSS, as shown in the nomogram. The larger the tumor, the higher the patient's risk of death. Second, surgery is the second most important factor affecting patient survival. Survival was most increased in patients with $\mathrm{PN}$, followed by RN and local tumor excision and was lowest in patients who did not undergo surgery. Tumor TNM stage and histological tumor grade are important risk factors for patient prognosis. Patients with higher TNM stage or histological grade had lower survival rates. In addition, the age of the patients was also a key factor; the older the patient, the greater the risk of death. Finally, married patients, women, and other races had a relatively high survival rate.

\section{Validation of the Nomogram}

In the training cohort and validation cohort, we first use the Cindex to validate the accuracy of the nomogram. The C-index of the training cohort and validation cohort was $0.81(95 \%$ CI: $0.802-0.818)$ and 0.818 (95\% CI: 0.806-0.830), respectively, indicating the accuracy and discrimination of the nomogram. In the training cohort and validation cohort, the calibration curve showed that the observed value of the prediction model was highly consistent with the predicted value, proving that the prediction model had good accuracy (Figure 3). The AUC of the training cohort and validation cohort also suggested that the prediction model had good accuracy (Figure 4). In the training cohort, the AUC of the prediction model was 86.8 (95\% CI, 85.588.0), 84.8 (95\% CI, 83.8-85.9), and 82.8 (95\% CI, 80.8-84.8) in 1 -, 3-, and 5 years, respectively. In the validation cohort, the AUC of the prediction model was 86.0 (95\% CI, 84.0-88.0), $85.0(95 \%$ CI, 83.5-86.4), and 83.3 (95\% CI, 81.6-85.0), respectively.
TABLE 1 | Clinicopathological characteristics of elderly patients with cCRCC.

\begin{tabular}{|c|c|c|c|c|}
\hline & $\begin{array}{c}\text { All } \\
N=33,509\end{array}$ & $\begin{array}{c}\text { Training } \\
\text { cohort } \\
N=23,412\end{array}$ & $\begin{array}{c}\text { validation } \\
\text { cohort } \\
N=10,097\end{array}$ & $p$ \\
\hline Age & 72.7 (5.91) & 72.7 (5.90) & 72.8 (5.95) & 0.515 \\
\hline Race & & & & 0.073 \\
\hline white & 29,034 (86.6\%) & 20,233 (86.4\%) & 8,801 (87.2\%) & \\
\hline black & 2,030 (6.06\%) & 1,463 (6.25\%) & 567 (5.62\%) & \\
\hline other & 2,445 (7.30\%) & 1,716 (7.33\%) & 729 (7.22\%) & \\
\hline Sex & & & & 0.313 \\
\hline Male & $20,201(60.3 \%)$ & $14,156(60.5 \%)$ & 6,045 (59.9\%) & \\
\hline Female & 13,308 (39.7\%) & 9,256 (39.5\%) & 4,052 (40.1\%) & \\
\hline Marriage & & & & 0.332 \\
\hline No & 12,270 (36.6\%) & 8,533 (36.4\%) & 3,737 (37.0\%) & \\
\hline Married & $21,239(63.4 \%)$ & 14,879 (63.6\%) & 6,360 (63.0\%) & \\
\hline $\begin{array}{l}\text { Year of } \\
\text { diagnosis }\end{array}$ & & & & 0.651 \\
\hline 2004-2010 & 13,295 (39.7\%) & 9,308 (39.8\%) & 3,987 (39.5\%) & \\
\hline 2010-2018 & $20,214(60.3 \%)$ & $14,104(60.2 \%)$ & $6,110(60.5 \%)$ & \\
\hline Laterality & & & & 0.138 \\
\hline Left & 16,447 (49.1\%) & $11,554(49.4 \%)$ & 4,893 (48.5\%) & \\
\hline Right & 17,062 (50.9\%) & $11,858(50.6 \%)$ & 5,204 (51.5\%) & \\
\hline Grade & & & & 0.760 \\
\hline I & 4,077 (12.2\%) & 2,834 (12.1\%) & 1,243 (12.3\%) & \\
\hline$\|$ & $17,480(52.2 \%)$ & $12,212(52.2 \%)$ & 5,268 (52.2\%) & \\
\hline III & 9,595 (28.6\%) & 6,698 (28.6\%) & 2,897 (28.7\%) & \\
\hline IV & 2,357 (7.03\%) & 1,668 (7.12\%) & 689 (6.82\%) & \\
\hline T & & & & 0.266 \\
\hline T1a & 14,019 (41.8\%) & 9,743 (41.6\%) & 4,276 (42.3\%) & \\
\hline $\mathrm{T} 1 \mathrm{~b}$ & 8,063 (24.1\%) & 5,694 (24.3\%) & 2,369 (23.5\%) & \\
\hline T2 & 2,987 (8.91\%) & 2,113 (9.03\%) & 874 (8.66\%) & \\
\hline T3 & 8,370 (25.0\%) & 5,811 (24.8\%) & 2,559 (25.3\%) & \\
\hline T4 & 70 (0.21\%) & $51(0.22 \%)$ & 19 (0.19\%) & \\
\hline$N$ & & & & 0.080 \\
\hline NO & 32,620 (97.3\%) & 22,815 (97.5\%) & 9,805 (97.1\%) & \\
\hline $\mathrm{N} 1$ & 889 (2.65\%) & 597 (2.55\%) & 292 (2.89\%) & \\
\hline M & & & & 0.362 \\
\hline MO & 31,320 (93.5\%) & $21,902(93.6 \%)$ & 9,418 (93.3\%) & \\
\hline M1 & 2,189 (6.53\%) & 1,510 (6.45\%) & 679 (6.72\%) & \\
\hline Tumor size & 51.8 (32.3) & 51.7 (31.0) & $52.0(35.0)$ & 0.449 \\
\hline Surgery & & & & 0.426 \\
\hline No & 1,227 (3.66\%) & 867 (3.70\%) & 360 (3.57\%) & \\
\hline $\begin{array}{l}\text { Local tumor } \\
\text { excision }\end{array}$ & 1,718 (5.13\%) & 1,173 (5.01\%) & 545 (5.40\%) & \\
\hline $\begin{array}{l}\text { Partial } \\
\text { nephrectomy }\end{array}$ & 8,791 (26.2\%) & 6,127 (26.2\%) & 2,664 (26.4\%) & \\
\hline $\begin{array}{l}\text { Radical } \\
\text { nephrectomy }\end{array}$ & 21,773 (65.0\%) & 15,245 (65.1\%) & 6,528 (64.7\%) & \\
\hline Chemotherapy & & & & 0.038 \\
\hline No/Unknown & 32,004 (95.5\%) & 22,397 (95.7\%) & 9,607 (95.1\%) & \\
\hline Yes & 1,505 (4.49\%) & 1,015 (4.34\%) & 490 (4.85\%) & \\
\hline Radiation: & & & & 0.354 \\
\hline No/Unknown & 32,869 (98.1\%) & 22,976 (98.1\%) & 9,893 (98.0\%) & \\
\hline Yes & 640 (1.91\%) & 436 (1.86\%) & 204 (2.02\%) & \\
\hline
\end{tabular}


TABLE 2 | Univariate and multivariate analyses of CSS in training cohort.

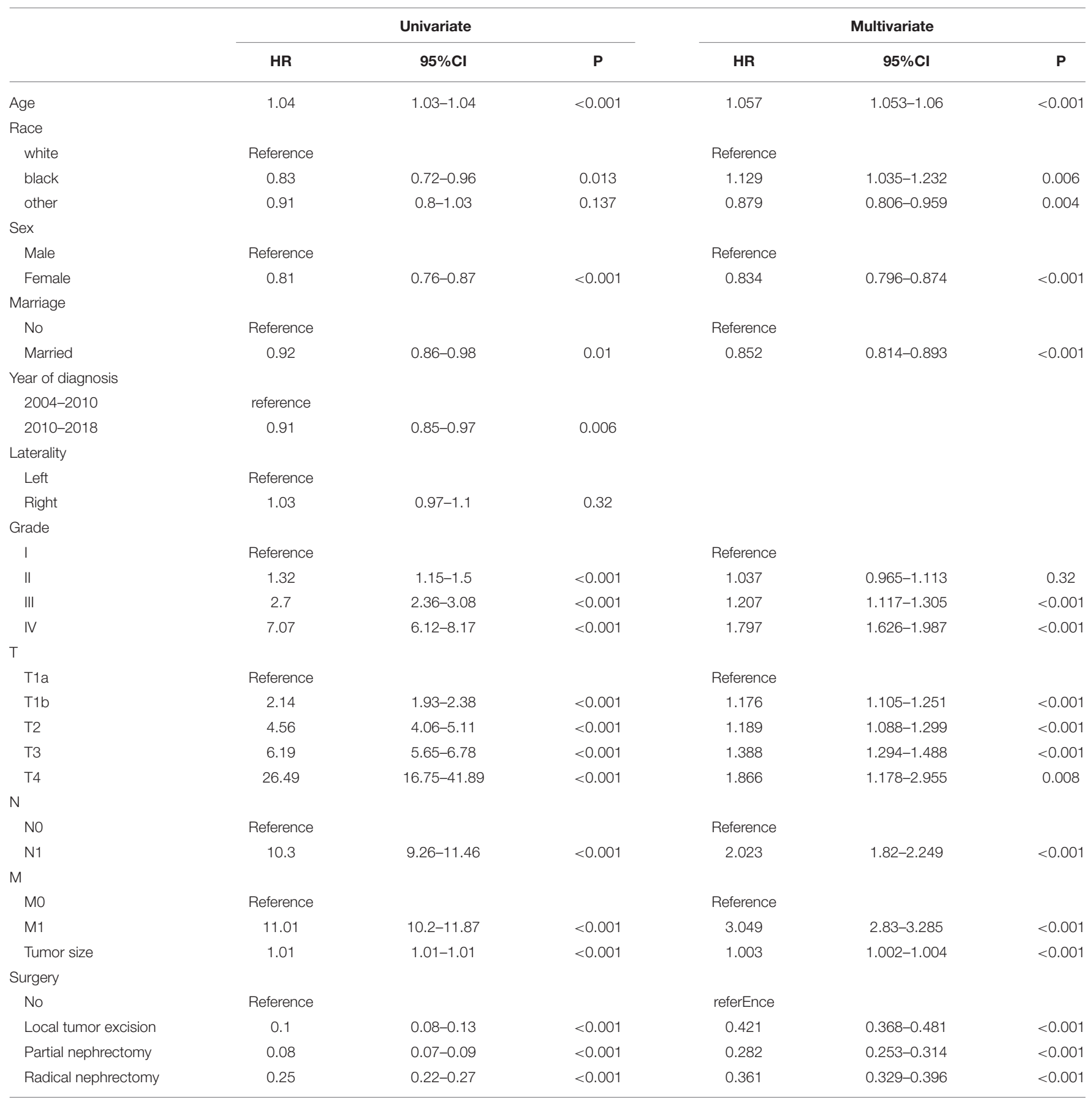

\section{Clinical Application of the Nomogram}

Decision curve analysis showed a good clinical application value (Figure 5). Moreover, DCA results showed that the DCA of the nomogram was significantly superior to the traditional TNM staging system in predicting patient survival. We divided the patients into the high-risk group (overall score $\leq 67.9$ ) and the low-risk group (overall score $>67.9$ ). The K-M curve showed that in the training cohort and validation cohort, the survival rate of patients in the high-risk group was significantly lower than that in the low-risk group (Figure 6). The 1-, 3-, and 5 years survival rates in high-risk group were 0.920 (95\% CI, $0.916-0.924$ ), 0.808 (95\% CI, 0.802-0.815), and 0.732 (95\% CI, 0.724-0.740), respectively. The 1-, 3-, and 5 years survival rates in the low-risk group were 0.992 (95\% CI, 0.991-0.994), 0.976 (95\% CI, 0.9730.979 ), and 0.954 ( $95 \% \mathrm{CI}, 0.950-0.957)$, respectively. Based on their risk grouping, we analyzed surgical options for patients in the low-risk and high-risk groups. In the low-risk group, we found that all patients received surgical treatment, and most 


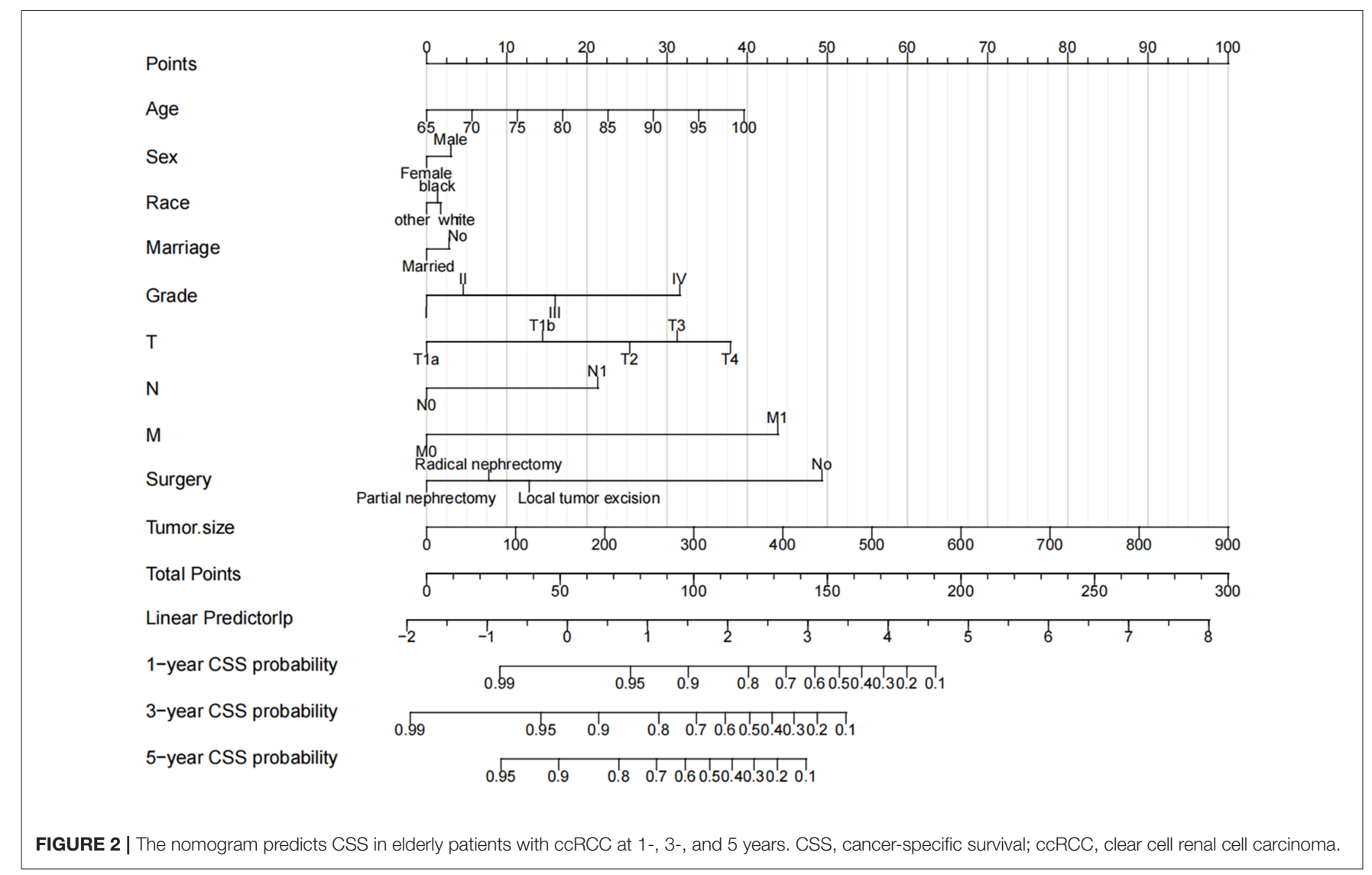

patients received $\mathrm{RN}$ and $\mathrm{PN}$, with significantly higher survival rates than local tumor excision. In the high-risk group, most patients who underwent $\mathrm{RN}$ had significantly higher survival rates than those who did not, with the highest survival rates among patients who underwent $\mathrm{PN}$ and local tumor excision (Figure 7).

\section{Online Application of Nomogram}

Based on our established programs, we developed a web application to predict 1-, 3-, 5 years CSS in elderly patients with ccRCC. Log on to https://liujiayan.shinyapps.io/DynNomapp/ can enter the website. The survival probability of patients can be obtained by inputting clinicopathological information of patients.

\section{DISCUSSION}

Renal cell carcinoma accounts for about $2 \%$ of all cancer diagnoses and deaths worldwide, with a higher incidence in developed countries and a severe burden on economic development. RCC is one of the top ten malignant tumors threatening human health (17). CcRCC is a category of RCC with a poor prognosis (7), and its risk factors are controversial. For example, Faramawi et al. (18) found that red meat consumption was an independent risk factor for the prognosis of ccRCC through meta-analysis, while Eun et al. (19) proved no clear correlation through 13 prospective studies. Similar conclusions also appeared when alcohol consumption (20) and exercise (21) were considered risk factors. Therefore, it is crucial to analyze and screen out specific risk factors, establish a predictive model, and accurately predict the prognosis of ccRCC patients.

Age is a critical factor in the occurrence and development of cancer, and so is ccRCC. With age, the probability of genetic mutations leading to cancer also increases. Aging is associated with highly reproducible DNA methylation changes, which helps to explain the higher prevalence of malignant tumors in the elderly (22). There is no consensus on defining the age of elderly patients, but more than $60 \%$ of initial cancer diagnoses and more than $70 \%$ of cancer deaths occur in patients over 65 years old (8). To improve the accuracy and representativeness of the prediction model, we included patients with ccRCC over 65 years old in this study. It is worth noting that newly diagnosed cases of ccRCC in the elderly are still mainly manifested by renal masses (8), and these masses usually show low-grade malignancy. However, clinicians mostly choose $\mathrm{RN}$ to avoid the poor prognosis caused by tumor metastasis, while such excessive treatment leads to more complications and even death in elderly patients $(23,24)$ and again highlights the crucial clinical value of this study.

Several studies have shown that married patients have increased cancer relative survival, even for untreated tumors (25-27). It may be related to the emotion of marriage and decision-making of medical treatment. Perini et al. (28) found 
A

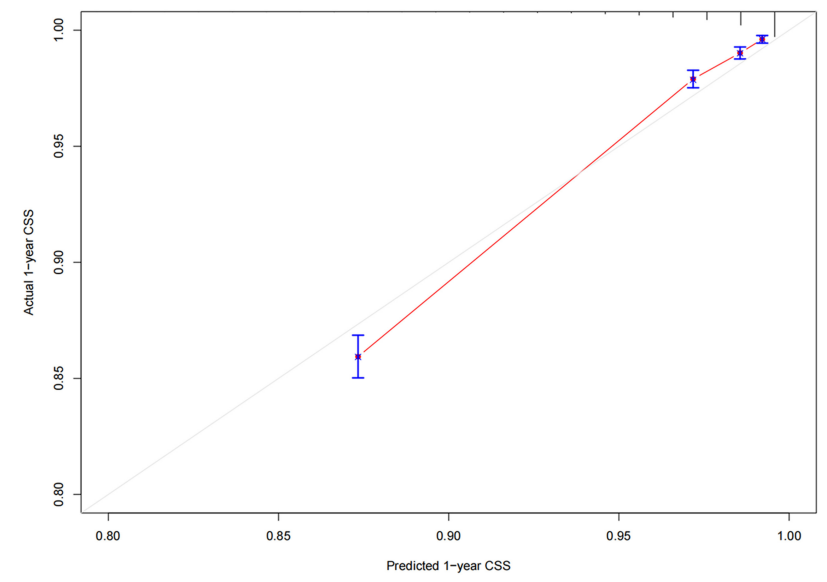

B

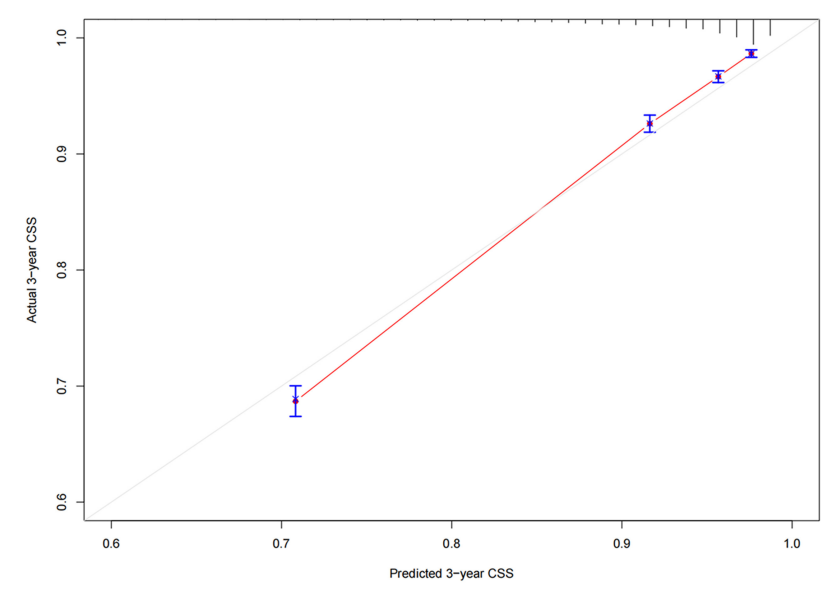

C

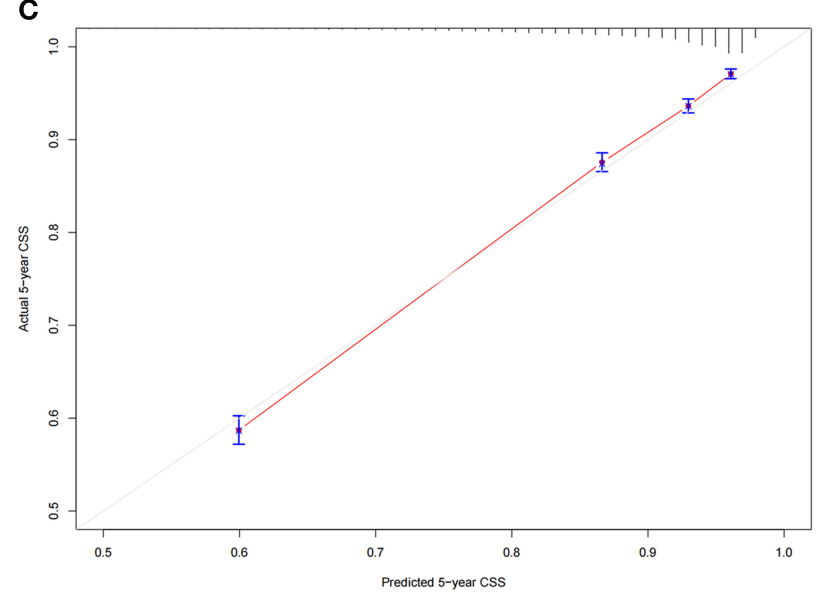

D

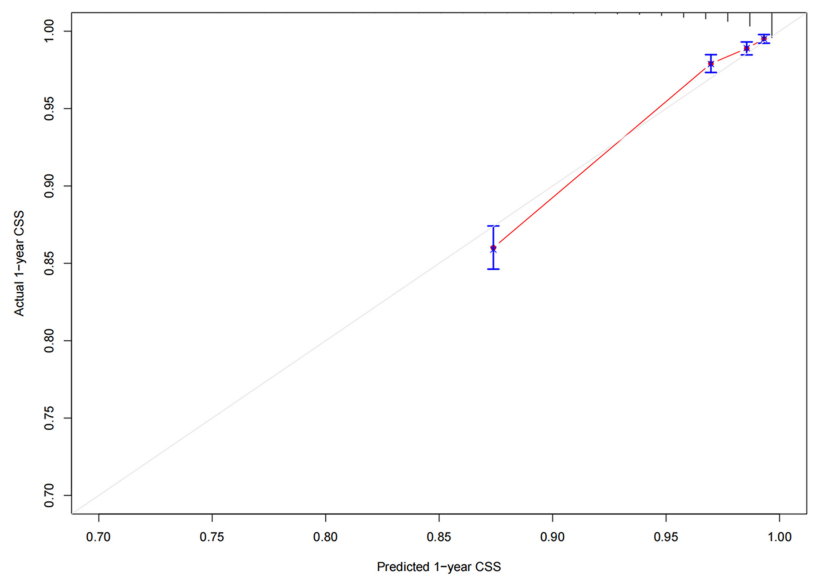

E

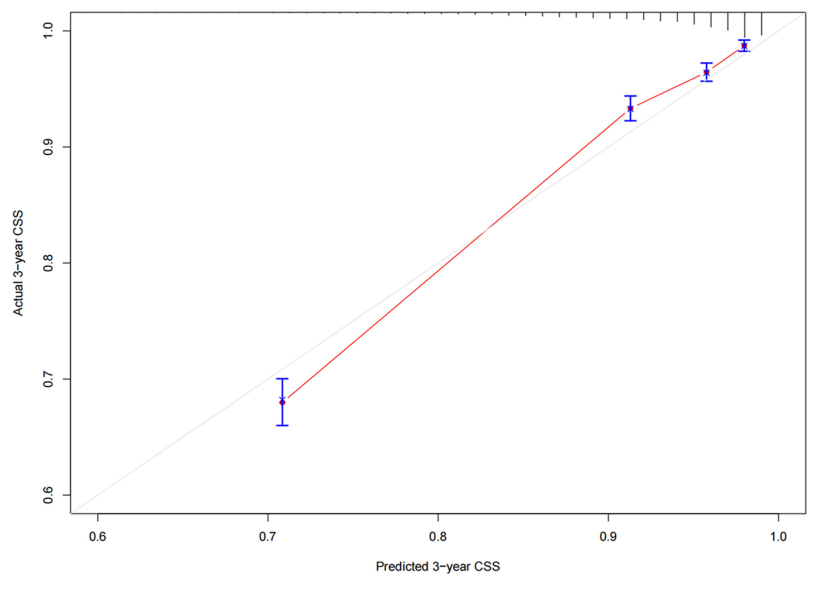

F

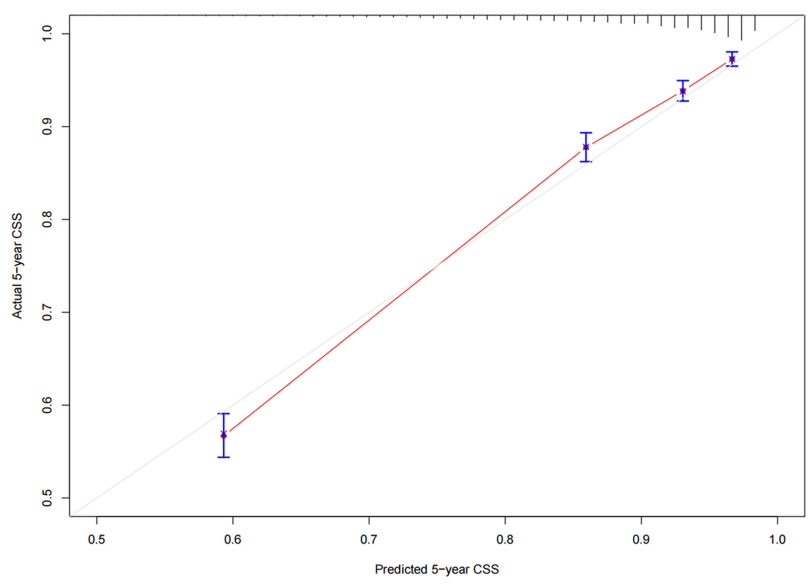

FIGURE 3 | Calibration curve of the nomogram. (A-C) Calibration curves of 1 -, 3 -, and 5 years CSS in the training cohort; (D-F) calibration curves of 1-, 3-, and 5 years CSS in the validation cohort. CSS, cancer-specific survival.

that depression was a risk factor for the low survival rate of cancer patients, while John et al. (29) found that the incidence of depression in men after marriage was significantly lower than that before marriage, which provided strong evidence for emotional factors. In addition, married cancer patients can detect physical abnormalities early and actively cooperate with 

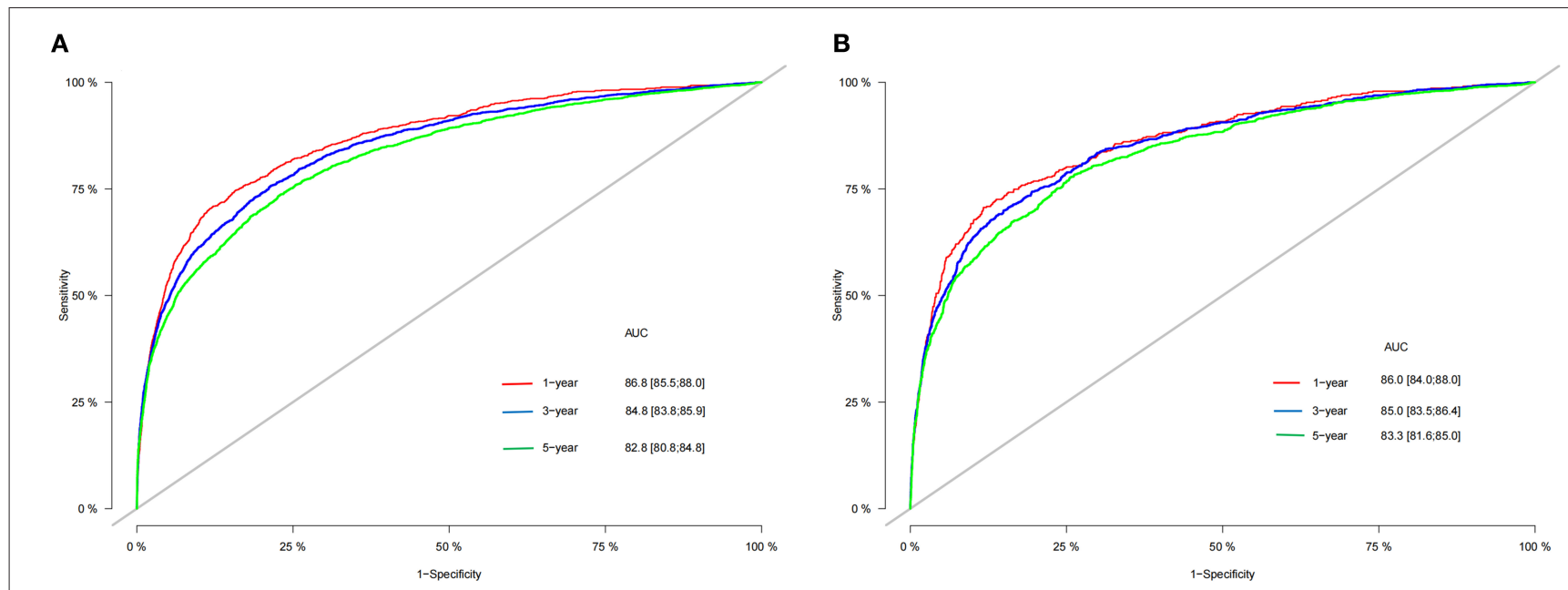

FIGURE 4 | AUC for predicting 1-, 3-, and 5 years CSS in training cohort (A) and validation cohort (B). CSS, cancer-specific survival; AUC, area under the receiver operator curve.
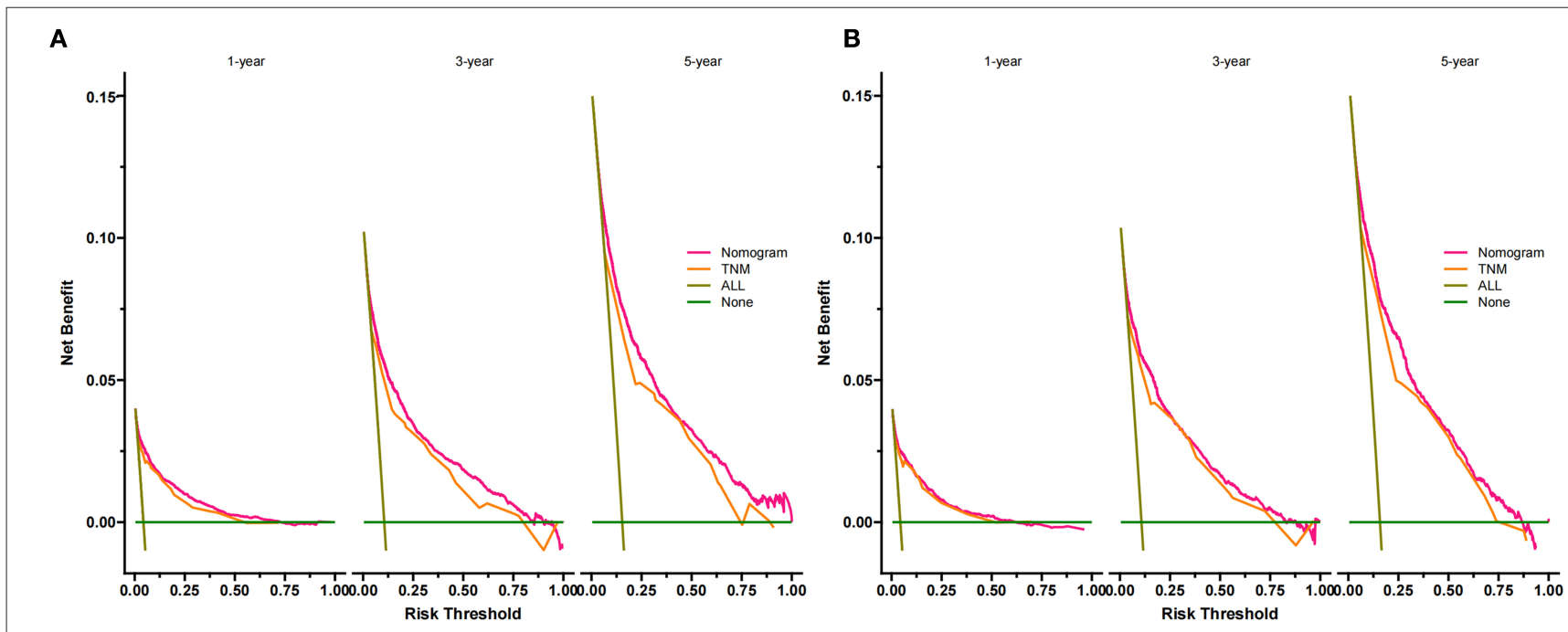

FIGURE 5 | DCA of the nomogram in the training cohort (A) and the validation cohort (B). The Y-axis represents a net benefit, and the X-axis represents threshold probability. The green line means no patients died, and the dark green line means all patients died. When the threshold probability is between 0 and $100 \%$, the net benefit of the model exceeds all deaths or none. DCA, decision curve analysis.

treatment, possibly out of the responsibility to the family after marriage (30). In conclusion, married patients showed significant improvement in RR compared with unmarried patients for cancer with a relatively low degree of malignancy, such as patients with ccRCC included in this study. Marriage is not beneficial for highly malignant cancers, such as pancreatic cancer and renal malignant rhabdomyoma tumor $(31,32)$.

On average, two individuals of the same sex are more than $99.9 \%$ alike. However, the genetic similarity between male and female individuals is only $98.5 \%$ (33). According to data from the SEER database, men tend to be diagnosed with RCC with higher stage and grade than women (34). Based on data analysis from the Multicenter Society for Renal Oncology Collaborative Study (CORONA) database, women's CSS (hazard ratio [HR] 0.75, $p$
$<0.001$ ) and overall survival (OS) (HR 0.80, $p<0.001$ ) were significantly better than those of men (35). In this study, the prognosis of female patients with ccRCC was also substantially better than that of male patients (HR: $0.834,95 \%$ CI: 0.796-0.874, $p<0.001$ ).

Classical RN involves removing the kidney, perirenal adipose tissue, adrenal gland, and local lymph nodes. It became the preferred treatment for ccRCC for some time. However, the massive loss of nephron caused by surgery can induce chronic kidney disease and cause death (36). Nevertheless, PN can preserve normal nephron to the maximum extent and is widely used in ccRCC cases with tumor sizes $<7 \mathrm{~cm}$ in length. Nowadays, the biggest challenge for PN is to avoid positive excision margin and reduce postoperative complications, such 

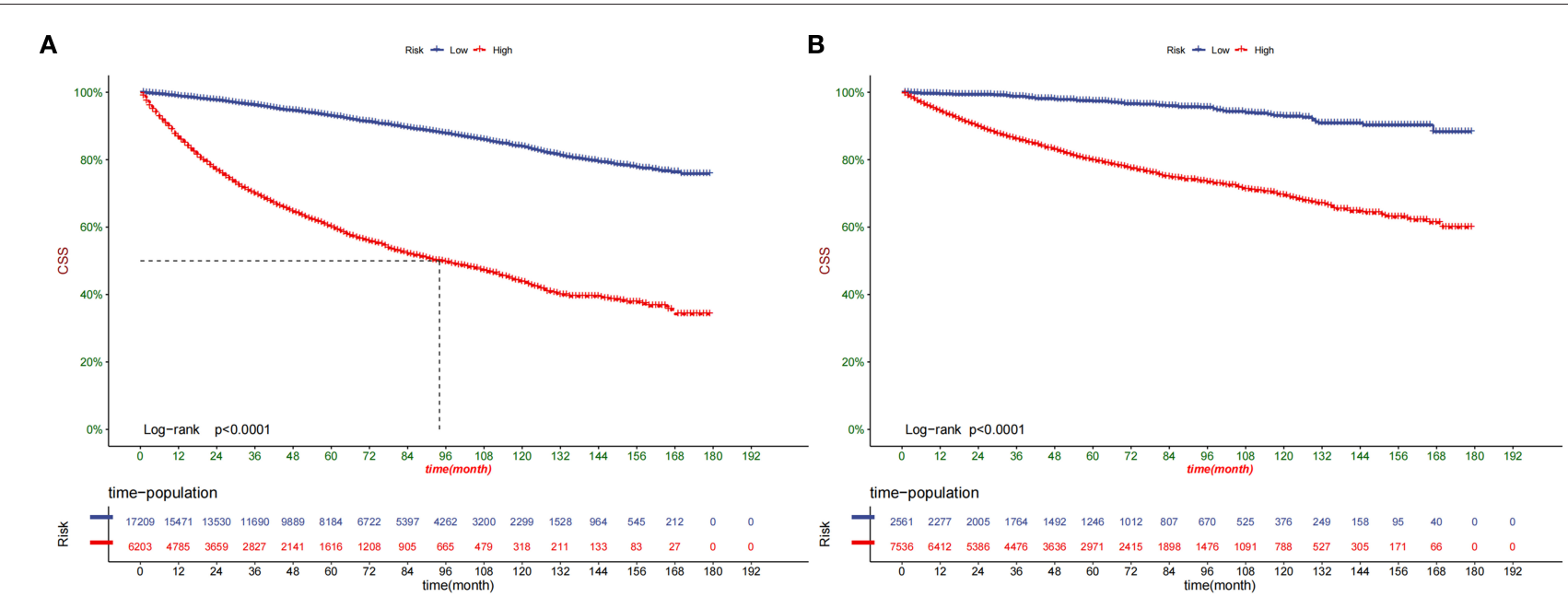

FIGURE 6 | Kaplan-Meier curves of patients in the low-risk and high-risk groups in training cohort (A) and validation cohort (B).

A

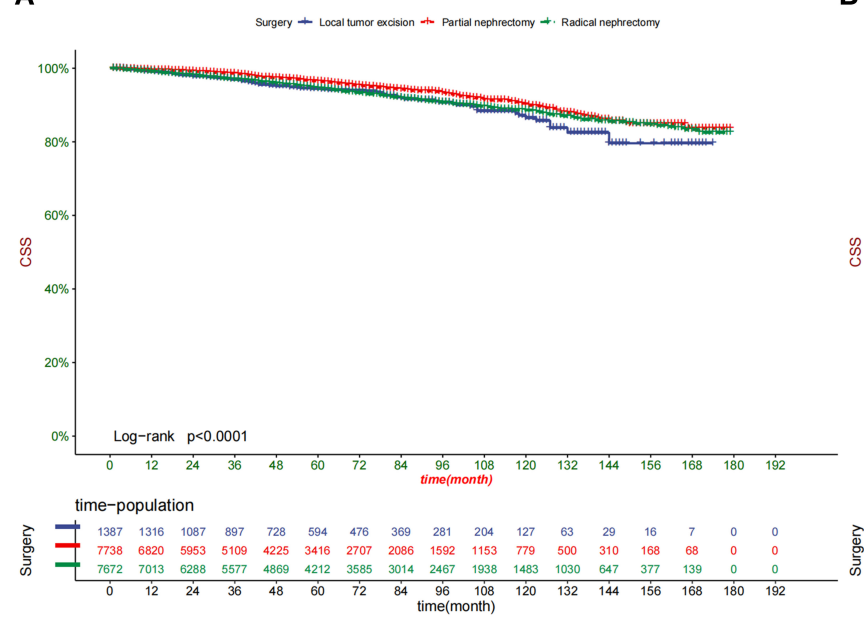

B

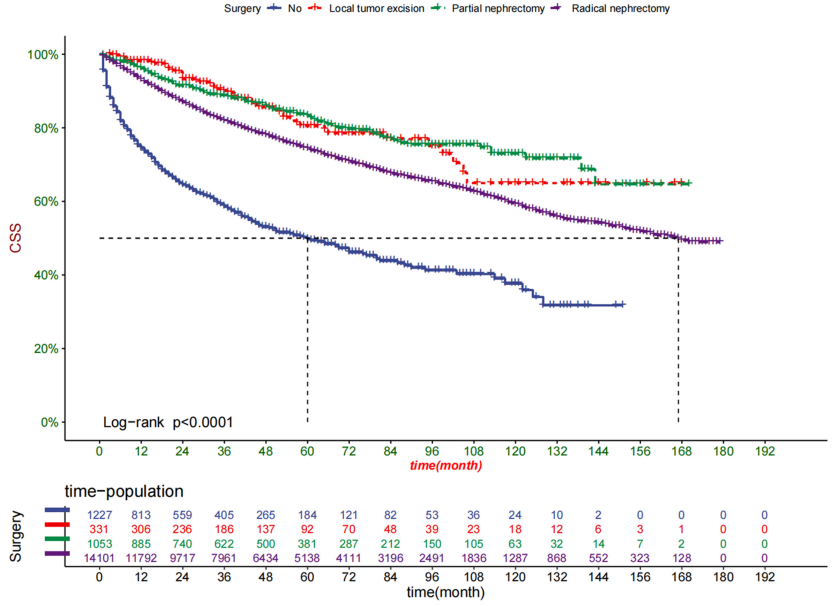

FIGURE 7 | Kaplan-Meier curves of patients with different surgery in the low-risk group (A) and high-risk group (B).

as hematuria, perirenal hematoma, and urinary fistula (37). Local tumor excision and active monitoring (AS) represented by RFA have been widely used in elderly patients and achieved good results due to contraindicated comorbidity and shortlife expectancy. In a meta-analysis of case studies, Dib et al. (38) showed that cryoablation and RFA efficacy were 89 and $90 \%$, respectively. In addition, RFA and other treatments can be operated under the guidance of ultrasound or CT positioning, effectively avoiding the damage of anesthetics to the heart, liver, and other organs of the elderly. Nevertheless, the surgical recurrence rate of local tumor resection is much higher than that of RN and PN (37). This study found that low-risk patients were more likely to benefit from PN and RN, while high-risk patients were more likely to benefit from $\mathrm{PN}$ and local tumor excision.

TNM staging of RCC was based on the 2021 European Association of Urology (EAU) updated criteria (39). The Fuhrman grading system was adopted for tumor classification.
According to WHO recommendations, grade I was defined as well differentiated, grade II as moderately differentiated, grade III as poorly differentiated, and grade IV as undifferentiated (36). We found that high TNM staging and histologic grade were associated with poor prognosis, which is consistent with the conclusions of previous studies (40).

In recent years, the broad application of the nomogram prediction model has gradually replaced the traditional TNM staging. First, the c-index of traditional TMN staging for prognosis prediction is about 0.6 , which is relatively inaccurate. Second, the prognosis of patients is also affected by many nonanatomic factors, such as age, gender, race, marital status, and surgical methods (41). However, the C-index of this study is 0.81 , which is of higher accuracy than the traditional TNM system. Nomogram is a data-based graphical computing tool that can estimate the risk of developing a disease based on staging systems, such as the American Joint Commission on Cancer (AJCC) and 
other key risk factors related to prognosis (42). Today, many kinds of nomograms have been developed and applied to RCC, such as Chen et al. (43), who included 6,105 elderly patients with metastatic RCC in the SEER database from 2010 to 2015, indicating the advantages of PN in elderly mRCC. Michele et al. (44) included 4,541 patients with RCC over 75 years of age in the SEER database from 2004 to 2014 and proved that PN did not increase the mortality of elderly patients. With the update of the SEER database and the improvement of patients' clinical information, this study included 33,509 elderly CCRCC patients from 2004 to 2018, which was superior to previous studies on elderly RCC in terms of the number of cases and c-index accuracy.

Still, there are some limitations to our study. First of all, our study was retrospective, and selection bias was inevitable. Secondly, some variables, such as patients' body mass index (BMI), smoking, drinking, heart disease, and other data cannot be obtained from the SEER database. However, we included significant variables, such as tumor stage, surgery, age, and other vital factors. Finally, our prediction model is only validated internally, and further external validation is necessary to validate the model's accuracy.

\section{CONCLUSION}

In this study, we explored prognostic factors in elderly patients with ccRCC. We found that age, sex, marriage, TNM stage, surgery, and tumor size were independent risk factors for CSS. We constructed a new nomogram to predict CSS in elderly patients with ccRCC with good accuracy and reliability, providing clinical guidance for patients and physicians.

\section{DATA AVAILABILITY STATEMENT}

The data analyzed in this study was obtained from the Surveillance, Epidemiology, and End Results (SEER) Program,

\section{REFERENCES}

1. Bray F, Ferlay J, Soerjomataram I, Siegel RL, Torre LA, Jemal A. Global cancer statistics 2018: GLOBOCAN estimates of incidence and mortality worldwide for 36 cancers in 185 countries [published correction appears in CA. Cancer J Clin. (2020). 70:313. doi: 10.3322/caac.21609

2. Quivy A, Daste A, Harbaoui A, Duc S, Bernhard JC, Gross-Goupil M, et al. Optimal management of renal cell carcinoma in the elderly: a review. Clin Interv Aging. (2013) 8:433-42. doi: 10.2147/CIA.S30765

3. Hsieh JJ, Purdue MP, Signoretti S, Swanton C, Albiges L, Schmidinger M, et al. Renal cell carcinoma. Nat Rev Dis Primers. (2017). 3:17009. doi: 10.1038/nrdp.2017.9

4. Siegel RL, Miller KD, Fuchs HE, Jemal A. Cancer Statistics, 2021. [published correction appears in CA. Cancer J Clin. (2021). 71:359. doi: 10.3322/caac.21654

5. Kim H, Shim BY, Lee SJ, Lee JY, Lee HJ, Kim IH. Loss of Von Hippel-Lindau (VHL) Tumor Suppressor Gene Function: VHL-HIF Pathway and Advances in Treatments for Metastatic Renal Cell Carcinoma (RCC). Int J Mol Sci. (2021) 22:9795. doi: 10.3390/ijms22189795

6. Jonasch E, Gao J, Rathmell WK. Renal cell carcinoma. BMJ. (2014) 349:g4797. doi: 10.1136/bmj.g4797 the following licenses/restrictions apply: To request access to the SEER Incidence Databases, you will first need to create a SEER*Stat Account and submit a request form. Requests to access these datasets should be directed to: https://service.cancer.gov/ seer-data-access. For questions regarding the data access request process, send an email to SEERDataAccess@mail.nih.gov.

\section{ETHICS STATEMENT}

Ethical review and approval was not required for the study on human participants in accordance with the local legislation and institutional requirements. Written informed consent from the patients/ participants or patients/participants' legal guardian/next of kin was not required to participate in this study in accordance with the national legislation and the institutional requirements.

\section{AUTHOR CONTRIBUTIONS}

JW and CZ designed the study. CZ, JW, JL, LJ, ML, and XT collected and analyzed the data. JW drafted the initial manuscript. CZ, TM, JL, ZZ, and DH revised the article critically. $\mathrm{CZ}, \mathrm{JL}, \mathrm{DH}, \mathrm{LJ}$, and $\mathrm{XT}$ reviewed and edited the article. All authors approved the final manuscript.

\section{FUNDING}

This study was supported by the Kunming City Health Science and Technology Talent 1000 training Project [no. 2020SW (Reserve)-112], Kunming Health and Health Commission Health Research Project (no. 2020-0201-001), Kunming Xishan District Science and Technology Project (no. 2020-Xike word 23), Kunming Medical Joint Project of Yunnan Science and Technology Department (no. 202001 AY070001-271), and Special Key Project of Chongqing Technology Innovation and Application Development (no. Cstc2019jscx-tjsbX0003).

7. Jonasch E, Walker CL, Rathmell WK. Clear cell renal cell carcinoma ontogeny and mechanisms of lethality. Nat Rev Nephrol. (2021) 17:24561. doi: 10.1038/s41581-020-00359-2

8. González León T, Morera Pérez M. Renal cancer in the elderly. Curr Urol Rep. (2016) 17:6. doi: 10.1007/s11934-015-0562-2

9. Motzer RJ, Banchereau R, Hamidi H, Powles T, McDermott D, Atkins $\mathrm{MB}$, et al. Molecular Subsets in Renal Cancer Determine Outcome to Checkpoint and Angiogenesis Blockade. Cancer Cell. (2020). 38:80317.e4. doi: 10.1016/j.ccell.2020.10.011

10. Labriola MK, Zhu J, Gupta RT, McCall S, Jackson J, Kong EF, et al. Characterization of tumor mutation burden, PD-L1 and DNA repair genes to assess relationship to immune checkpoint inhibitors response in metastatic renal cell carcinoma. J Immunother Cancer. (2020) 8:e000319. doi: 10.1136/jitc-2019-000319

11. Murakami T, Tanaka N, Takamatsu K, Hakozaki K, Fukumoto K, Masuda $\mathrm{T}$, et al. Multiplexed single-cell pathology reveals the association of CD8 Tcell heterogeneity with prognostic outcomes in renal cell carcinoma. Cancer Immunol Immunother. (2021) 70:3001-13. doi: 10.1007/s00262-021-03006-2

12. Haddad H, Rini BI. Current treatment considerations in metastatic renal cell carcinoma. Curr Treat Options Oncol. (2012) 13:212-29. doi: 10.1007/s11864-012-0182-8 
13. Parker WP, Cheville JC, Frank I, Zaid HB, Lohse CM, Boorjian SA, et al. Application of the stage, size, grade, and necrosis (SSIGN) score for clear cell renal cell carcinoma in contemporary patients. Eur Urol. (2017) 71:66573. doi: 10.1016/j.eururo.2016.05.034

14. Beisland C, Gudbrandsdottir G, Reisæter LA, Bostad L, WentzelLarsen T, Hjelle KM. Contemporary external validation of the Leibovich model for prediction of progression after radical surgery for clear cell renal cell carcinoma. Scand J Urol. (2015) 49:205-10. doi: 10.3109/21681805.2014.980844

15. Awais M, Ghayvat H, Krishnan Pandarathodiyil A, Nabillah Ghani WM, Ramanathan A, Pandya S, et al. Healthcare professional in the loop (HPIL): classification of standard and oral cancer-causing anomalous regions of oral cavity using textural analysis technique in autofluorescence imaging. Sensors (Basel). (2020) 20:5780. doi: 10.3390/s20205780

16. Shah A, Ahirrao S, Pandya S, Kotecha K, Rathod S. Smart cardiac framework for an early detection of cardiac arrest condition and risk. Front Public Health. (2021) 9:762303. doi: 10.3389/fpubh.2021.762303

17. Sharma R. An examination of colorectal cancer burden by socioeconomic status: evidence from GLOBOCAN (2018) EPMA J. (2019). 11:95117. doi: 10.1007/s13167-019-00185-y

18. Faramawi MF, Johnson E, Fry MW, Sall M, Zhou Y. Consumption of different types of meat and the risk of renal cancer: meta-analysis of case-control studies [published correction appears in Cancer Causes Control. Cancer Causes Control. (2007). 18:125-33. doi: 10.1007/s10552-006-0104-9

19. Lee JE, Spiegelman D, Hunter DJ, Albanes D, Bernstein L, van den Brandt $\mathrm{PA}$, et al. Fat, protein, and meat consumption and renal cell cancer risk: a pooled analysis of 13 prospective studies. J Natl Cancer Inst. (2008) 100:1695706. doi: $10.1093 /$ jnci/djn386

20. Cohortiawan VW, Stram DO, Nomura AM, Kolonel LN, Henderson BE. Risk factors for renal cell cancer: the multiethnic cohort. Am J Epidemiol. (2007) 166:932-40. doi: 10.1093/aje/kwm170

21. Bergström A, Terry P, Lindblad P, Lichtenstein P, Ahlbom A, Feychting M, et al. Physical activity and risk of renal cell cancer. Int J Cancer. (2001) 92:1557. doi: 10.1002/1097-0215(200002)9999:9999\&lt;::AID-IJC1162\&gt;3.0.CO;2$\mathrm{S}$

22. Lin Q, Wagner W. Epigenetic aging signatures are coherently modified in cancer. PLoS Genet. (2015) 11:e1005334. doi: 10.1371/journal.pgen.1005334

23. Escudier B, Porta C, Schmidinger M, Rioux-Leclercq N, Bex A, Khoo $\mathrm{V}$, et al. Renal cell carcinoma: ESMO Clinical Practice Guidelines for diagnosis, treatment and follow-up ${ }^{\dagger}$. Ann Oncol. (2019) 30:706720. doi: 10.1093/annonc/mdz056

24. Gontero P, Joniau S, Oderda M, Ruutu M, Van Poppel H, Laguna MP, et al. Active surveillance for small renal tumors: have clinical concerns been addressed so far? Int J Urol. (2013) 20:356-61. doi: 10.1111/j.1442-2042.2012.03227.x

25. Li Z, Wang K, Zhang X, Wen J. Marital status and survival in patients with rectal cancer: A population-based STROBE cohort study [published correction appears in Medicine (Baltimore). (2018). 97:e10872. Medicine (Baltimore). (2018) 97:e0637. doi: 10.1097/MD.0000000000010637

26. Gomez SL, Hurley S, Canchola AJ, Keegan TH, Cheng I, Murphy JD, et al. Effects of marital status and economic resources on survival after cancer: A population-based study. Cancer. (2016) 122:1618-25. doi: 10.1002/cncr.29885

27. Martínez ME, Anderson K, Murphy JD, Hurley S, Canchola AJ, Keegan $\mathrm{TH}$, et al. Differences in marital status and mortality by race/ethnicity and nativity among California cancer patients. Cancer. (2016) 122:15708. doi: $10.1002 /$ cncr. 29886

28. Bortolato B, Hyphantis TN, Valpione S, Perini G, Maes M, Morris G, et al. Depression in cancer: The many biobehavioral pathways driving tumor progression. Cancer Treat Rev. (2017) 52:58-70. doi: 10.1016/j.ctrv.2016.11.004

29. St John PD, Montgomery PR. Marital status, partner satisfaction, and depressive symptoms in older men and women. Can J Psychiatry. (2009) 54:487-92. doi: 10.1177/070674370905400710

30. Enewold L, Harlan LC, Tucker T, McKenzie S. Pancreatic cancer in the USA: persistence of undertreatment and poor outcome. J Gastrointest Cancer. (2015) 46:9-20. doi: 10.1007/s12029-014-9668-x

31. Zhanghuang C, Chen S, Li L, Yang Z, Xie Y, Li J, et al. Clinical and molecular differentiation between malignant rhabdoid tumor of the kidney and normal tissue: a two-case report. Front Oncol. (2021) 11:659709. doi: 10.3389/fonc.2021.659709

32. Merrill RM, Johnson E. Benefits of marriage on relative and conditional relative cancer survival differ between males and females in the USA. J Cancer Surviv. (2017) 11:578-89. doi: 10.1007/s11764-017-0627-y

33. Lucca I, Klatte T, Fajkovic H, de Martino M, Shariat SF. Gender differences in incidence and outcomes of urothelial and kidney cancer. Nat Rev Urol. (2015) 12:653. doi: 10.1038/nrurol.2015.232

34. Aron M, Nguyen MM, Stein RJ, Gill IS. Impact of gender in renal cell carcinoma: an analysis of the SEER database. Eur Urol. (2008) 54:13340. doi: 10.1016/j.eururo.2007.12.001

35. May M, Aziz A, Zigeuner R, Chromecki T, Cindolo L, Schips L, et al. Gender differences in clinicopathological features and survival in surgically treated patients with renal cell carcinoma: an analysis of the multicenter CORONA database. World J Urol. (2013) 31:1073-80. doi: 10.1007/s00345-013-1071-x

36. Borgmann H, Musquera M, Haferkamp A, Vilaseca A, Klatte T, Shariat SF, et al. Prognostic significance of Fuhrman grade and age for cancer-specific and overall survival in patients with papillary renal cell carcinoma: results of an international multi-institutional study on 2189 patients. World J Urol. (2017) 35:1891-7. doi: 10.1007/s00345-017-2078-5

37. MacLennan S, Imamura M, Lapitan MC, Omar MI, Lam TB, HilvanoCabungcal AM, et al. Systematic review of perioperative and quality-of-life outcomes following surgical management of localised renal cancer. Eur Urol. (2012) 62:1097-117. doi: 10.1016/j.eururo.2012.07.028

38. El Dib R, Touma NJ, Kapoor A. Cryoablation vs radiofrequency ablation for the treatment of renal cell carcinoma: a meta-analysis of case series studies. BJU Int. (2012) 110:510-6. doi: 10.1111/j.1464-410X.2011.10885.x

39. Rouprêt M, Babjuk M, Burger M, Capoun O, Cohen D, Compérat EM, et al. European Association of Urology Guidelines on Upper Urinary Tract Urothelial Carcinoma: (2020). Update Eur Urol. (2021) 79:6279. doi: 10.1016/j.eururo.2020.05.042

40. Hofbauer SL, de Martino M, Seemann C, Zamani N, Lucca I, Haitel A, et al. Associations between presenting symptoms, clinicopathological parameters, and prognosis in a contemporary series of patients with renal cell carcinoma. Korean J Urol. (2014) 55:505-10. doi: 10.4111/kju.2014.55.8.505

41. Serdy KM, Leone JP, Dabbs DJ, Bhargava R. Male breast cancer. Am J Clin Pathol. (2017) 147:110-9. doi: 10.1093/ajcp/aqw207

42. Liu X, Wu Z, Lin E, Li W, Chen Y, Sun X, et al. Systemic prognostic score and nomogram based on inflammatory, nutritional and tumor markers predict cancer-specific survival in stage II-III gastric cancer patients with adjuvant chemotherapy. Clin Nutr. (2019) 38:1853-60. doi: 10.1016/j.clnu.2018.07.015

43. Chen J, He Q, Liu W, Li Y, Zhuang W. The effect of cytoreductive partial nephrectomy in elderly patients with metastatic renal cell carcinoma. Clin Interv Aging. (2020) 15:431-9. doi: 10.2147/CIA.S243902

44. Marchioni M, Preisser F, Bandini M, Nazzani S, Tian Z, Kapoor A, et al. Comparison of partial versus radical nephrectomy effect on other-cause mortality, cancer-specific mortality, and 30-day mortality in patients older than 75 years. Eur Urol Focus. (2019) 5:467-73. doi: 10.1016/j.euf.2018. 01.007

Conflict of Interest: The authors declare that the research was conducted in the absence of any commercial or financial relationships that could be construed as a potential conflict of interest.

Publisher's Note: All claims expressed in this article are solely those of the authors and do not necessarily represent those of their affiliated organizations, or those of the publisher, the editors and the reviewers. Any product that may be evaluated in this article, or claim that may be made by its manufacturer, is not guaranteed or endorsed by the publisher.

Copyright (C) 2022 Zhanghuang, Wang, Zhang, Jin, Tan, Mi, Liu, Li and He. This is an open-access article distributed under the terms of the Creative Commons Attribution License (CC BY). The use, distribution or reproduction in other forums is permitted, provided the original author(s) and the copyright owner(s) are credited and that the original publication in this journal is cited, in accordance with accepted academic practice. No use, distribution or reproduction is permitted which does not comply with these terms. 